\title{
Fractional order mixed difference operator and its applications in angular approximation
}

\author{
Ramazan Akgün (D) \\ Balikesir University, Faculty of Arts and Sciences, Department of Mathematics, Cagis Yerleskesi, 10145, \\ Balikesir, Turkey
}

\begin{abstract}
Lebesgue spaces are considered with Muckenhoupt weights. Fractional order mixed difference operator is investigated to obtain mixed fractional modulus of smoothness in these spaces. Using this modulus of smoothness we give the proof of direct and inverse estimates of angular trigonometric approximation. Also we obtain an equivalence between fractional mixed modulus of smoothness and fractional mixed $K$-functional.
\end{abstract}

Mathematics Subject Classification (2010). 42A10, 41A17, 41A27, 41A28

Keywords. direct theorem, inverse theorem, Muckenhoupt weights, modulus of smoothness

\section{Introduction}

Let $\mathbb{T}:=[0,2 \pi], \mathbb{T}^{2}:=\mathbb{T} \times \mathbb{T}$, and $L_{\omega}^{p}:=L_{\omega}^{p}\left(\mathbb{T}^{2}\right)$ be the weighted Lebesgue space of functions $f(x, y): \mathbb{T}^{2} \rightarrow \mathbb{R}, 2 \pi$-periodic with respect to each variable $x, y$, and

$$
\|f\|_{p, \omega}^{p}:=\int_{\mathbb{T}^{2}}|f(x, y)|^{p} \omega(x, y) d x d y<\infty
$$

A function $\omega: \mathbb{T}^{2} \rightarrow[0, \infty)$ is called a weight on $\mathbb{T}^{2}$ if $\omega(x, y)$ is measurable and positive almost everywhere on $\mathbb{T}^{2}$. We denote by $A_{p}:=A_{p}\left(\mathbb{T}^{2}, \mathbb{J}\right), 1<p<\infty$, the collection of locally integrable weight functions $\omega$ such that $\omega(x, y)$ is $2 \pi$-periodic with respect to each variable $x, y$ and

$$
C:=\sup _{G \in \mathbb{J}} \frac{1}{|G|} \int_{G} \omega(x, y) d x d y\left(\frac{1}{|G|} \int_{G}[\omega(x, y)]^{\frac{-1}{p-1}} d x d y\right)^{p-1}<\infty,
$$

where $\mathbb{J}$ is the set of rectangles in $\mathbb{T}^{2}$ with sides parallel to coordinate axes. Least constant $C$ in (1.1) will be called the Muckenhoupt's constant of $\omega$ and denoted by $[\omega]_{A_{p}}$. In the present paper we considered approximation properties of the two dimensional Fourier series in Lebesgue spaces $L_{\omega}^{p}$ with weights $\omega$ belonging to the Muckenhoupt's class $A_{p}$. We consider a weighted mixed modulus of smoothness and obtain several angular trigonometric approximation inequalities involving angular trigonometric approximation errors and modulus of smoothness in $L_{\omega}^{p}$ with $\omega \in A_{p}, 1<p<\infty$.

Email address: rakgun@balikesir.edu.tr

Received: 23.05.2019; Accepted: 27.11.2019 
In the nonweighted case, on $\mathbb{T}^{2}$, with the classical nonweighted mixed modulus of smoothness of functions given in the classical Lebesgue spaces $L^{p}:=L^{p}\left(\mathbb{T}^{2}\right)$, approximation by "angle" were investigated by several mathematicians. One can see the papers ([16-20]) written by M. K. Potapov. Also in the works [22,24], transformed Fourier series and mixed modulus of smoothness were investigated. Embedding problems of the BesovNikolskii and Weyl-Nikolskii classes are studied in [21,23]. Ul'yanov type inequalities were considered in [25] and [26]. Mixed $K$-functionals were considered by C. Cottin in [8]. For the univariate case one can see the papers [10,12-14,29].

In the weighted spaces, generally, ordinary translation is not suitable to construct difference operator and modulus of smoothness. The modulus of smoothness defined here is applicable in some weighted spaces.

Let

$$
Y_{m_{1}, m_{2}}(f)_{p, \omega}=\inf _{T_{i}}\left\{\left\|f-\sum_{i=1}^{2} T_{i}\right\|_{p, \omega}: T_{i} \in \mathcal{T}_{m_{i}}\right\},
$$

where $\mathcal{T}_{m_{i}}$ is the set of all two dimensional trigonometric polynomial of degree at most $m_{i}$ with respect to variable $x_{i}(i=1,2)$.

Define the following Steklov averages

$$
\begin{gathered}
\sigma_{h, \circ} f(x, y):=\frac{1}{h} \int_{x-h / 2}^{x+h / 2} f(t, y) d t, \quad \sigma_{\circ, k} f(x, y):=\frac{1}{k} \int_{y-k / 2}^{y+k / 2} f(x, \tau) d \tau, \\
\sigma_{h, k} f(x, y):=\frac{1}{h k} \int_{x-h / 2}^{x+h / 2} \int_{y-k / 2}^{y+k / 2} f(t, \tau) d t d \tau \\
\sigma_{0, \circ} f(x, y)=\sigma_{\circ, 0} f(x, y)=\sigma_{0,0} f(x, y):=f(x, y) .
\end{gathered}
$$

Let $x, y \in \mathbb{T}, r, h, k>0, p \in(1, \infty), \omega \in A_{p}$, and $f \in L_{\omega}^{p}$. Define the quantities

$$
\begin{aligned}
& \nabla_{h, \circ}^{r, \circ} f(x, \cdot):=\left(\mathbb{I}-\sigma_{h, \circ}\right)^{r} f(x, \cdot)=\sum_{i=0}^{\infty}\left(\begin{array}{c}
r \\
i
\end{array}\right)(-1)^{i}\left(\sigma_{h, \circ} f\right)^{i}(x, \cdot) \\
& \nabla_{\circ, k}^{\circ, r} f(\cdot, y):=\left(\mathbb{I}-\sigma_{\circ, k}\right)^{r} f(\cdot, y)=\sum_{j=0}^{\infty}\left(\begin{array}{c}
r \\
j
\end{array}\right)(-1)^{j}\left(\sigma_{\circ, k} f\right)^{j}(\cdot, y) \\
& \nabla_{h, k}^{r, r} f(x, y):=\nabla_{h, \circ}^{r, \circ}\left(\nabla_{\circ, k}^{\circ, r} f\right)(x, y)
\end{aligned}
$$

where $\mathbb{I}$ is identity operator on $\mathbb{T}^{2},\left(\begin{array}{l}r \\ j\end{array}\right):=\frac{r(r-1) \ldots(r-j+1)}{j !}$ for $j>1,\left(\begin{array}{l}r \\ 1\end{array}\right):=r$ and $\left(\begin{array}{l}r \\ 0\end{array}\right):=1$ are binomial coefficients.

Definition 1.1. The fractional weighted mixed modulus of smoothness of $f \in L_{\omega}^{p}, 1<$ $p<\infty, \omega \in A_{p}, r \in\{0\} \cup \mathbb{R}^{+}$, defined as

$$
\Omega_{r}\left(f, \delta_{1}, \delta_{2}\right)_{p, \omega}= \begin{cases}\sup _{h, k}\left\{\left\|\nabla_{h, k}^{r, r} f\right\|_{p, \omega}: 0 \leq h \leq \delta_{1}, 0 \leq k \leq \delta_{2}\right\} & , r>0, \\ \|f\|_{p, \omega} & , r=0 .\end{cases}
$$

In the present work we obtain main properties of the weighted fractional order mixed modulus of smoothness (1.5). The first of them is given in following approximation error estimate (direct theorem of angular trigonometric approximation): 
Theorem 1.2. If $1<p<\infty, \omega \in A_{p}, f \in L_{\omega}^{p}$ and $r \in \mathbb{R}^{+}$, then there exists a constant $C_{[\omega]_{A_{p}}, p, r}$ depending only on Muckenhoupt's constant $[\omega]_{A_{p}}$ of $\omega$ and $p, r$ such that

$$
Y_{m_{1}, m_{2}}(f)_{p, \omega} \leq C_{[\omega]_{A_{p}}, p, r} \Omega_{r}\left(f, \frac{1}{m_{1}}, \frac{1}{m_{2}}\right)_{p, \omega}
$$

holds for $m_{1}, m_{2} \in \mathbb{N}$.

We use the notation for fractional Weyl type derivatives $f^{(r, s)}:=\frac{\partial^{r+s} f}{\partial x^{r} \partial y^{s}} ; f^{(r, \circ)}:=\frac{\partial^{r} f}{\partial x^{r}}$; $f^{(\circ, s)}:=\frac{\partial^{s} f}{\partial y^{s}}$. Let $W_{p, \omega}^{r, s}, r, s \in \mathbb{N}$, (respectively $W_{p, \omega}^{r, o} ; \quad W_{p, \omega}^{o, s}$ ) be denote the collection of integrable functions $f$ with $f^{(r, s)} \in L_{\omega}^{p}$ (respectively $f^{(r, \circ)} \in L_{\omega}^{p} ; \quad f^{(\circ, s)} \in L_{\omega}^{p}$ ).

Definition 1.3. The quantity

$$
\begin{aligned}
K(f, \delta, \xi, p, \omega, r, s):= & \inf _{g_{1}, g_{2}, g}\left\{\left\|f-g_{1}-g_{2}-g\right\|_{p, \omega}+\delta^{r}\left\|\frac{\partial^{r} g_{1}}{\partial x^{r}}\right\|_{p, \omega}+\right. \\
& \left.+\xi^{s}\left\|\frac{\partial^{s} g_{2}}{\partial y^{s}}\right\|_{p, \omega}+\delta^{r} \xi^{s}\left\|\frac{\partial^{r+s} g}{\partial x^{r} \partial y^{s}}\right\|_{p, \omega}\right\}
\end{aligned}
$$

is known as weighted mixed $K$-functional, where infimum is taken over $g_{1}, g_{2}, g$ so that $g_{1} \in W_{p, \omega}^{r, \circ}, g_{2} \in W_{p, \omega}^{\circ, s}, g \in W_{p, \omega}^{r, s}$, where $r, s \in \mathbb{R}^{+}:=(0, \infty), 1<p<\infty, \omega \in A_{p}, f \in L_{\omega}^{p}$.

By Theorem 1.2 we get the following equivalence between $\Omega_{r}\left(f, \delta_{1}, \delta_{2}\right)_{p, \omega}$ and mixed $K$-functional.

Theorem 1.4. If $1<p<\infty, \omega \in A_{p}, f \in L_{\omega}^{p}$, and $r \in \mathbb{R}^{+}$, then there exist constants $c_{[\omega]_{A_{p}}, p, r}>0, C_{[\omega]_{A_{p}}, p, r}>0$, depending only on Muckenhoupt's constant $[\omega]_{A_{p}}$ of $\omega$ and $p, r$, so that the equivalence

$$
\Omega_{r}\left(f, \delta_{1}, \delta_{2}\right)_{p, \omega} \leq C_{[\omega]_{A_{p}}, p, r} K\left(f, \delta_{1}, \delta_{2}, p, \omega, 2 r\right) \leq c_{[\omega]_{A_{p}}, p, r} \Omega_{r}\left(f, \delta_{1}, \delta_{2}\right)_{p, \omega}
$$

holds for $\delta_{1}, \delta_{2} \geq 0$.

Theorem 1.4 gives the following corollary.

Corollary 1.5. If $1<p<\infty, \omega \in A_{p}, f \in L_{\omega}^{p}$, and $r \in \mathbb{R}^{+}$then, there exist constants depending only on $[\omega]_{A_{p}}$ and $p, r$ such that

$$
\begin{gathered}
\Omega_{r}(f, \lambda \delta, \eta \xi)_{p, \omega} \leq c(1+\lfloor\lambda\rfloor)^{2 r}(1+\lfloor\eta\rfloor)^{2 r} \Omega_{r}(f, \delta, \xi)_{p, \omega}, \quad \delta, \xi>0, \\
\frac{\Omega_{r}\left(f, \delta_{1}, \delta_{2}\right)_{p, \omega}}{\delta_{1}^{2 r} \delta_{2}^{2 r}} \leq C \frac{\Omega_{r}\left(f, t_{1}, t_{2}\right)_{p, \omega}}{t_{1}^{2 r} t_{2}^{2 r}} \quad \text { for } 0<t_{i} \leq \delta_{i} ; \quad i=1,2
\end{gathered}
$$

where $\lfloor x\rfloor:=\max \{z \in \mathbb{Z}: z \leq x\}$.

Converse estimate to (1.6) is given in the next theorem.

Theorem 1.6. If $1<p<\infty, \omega \in A_{p}, f \in L_{\omega}^{p}$ and $r \in \mathbb{R}^{+}$, then there exist constants depending only on $[\omega]_{A_{p}}$ and $p, r$ so that

$$
\Omega_{r}\left(f, \frac{1}{m_{1}}, \frac{1}{m_{2}}\right)_{p, \omega} \leq \frac{C_{[\omega]_{A_{p}}, p, r}}{\prod_{i=1}^{2} m_{i}^{2 r}} \sum_{l_{i_{1}}=0}^{m_{1}} \sum_{l_{i_{2}}=0}^{m_{2}} \prod_{j=1}^{2}\left[\left(l_{i_{j}}+1\right)\right]^{2 r-1} Y_{l_{i_{1}}, l_{i_{2}}}(f)_{p, \omega} .
$$

In this paper, we will denote positive constant $C_{u, v, \ldots}$, depending only on the parameters $u, v, \ldots$ so that it can be different in different places. 


\section{Preliminary definition and results}

Suppose that $L^{1}$ is the collection of the Lebesgue integrable functions $f(x, y): \mathbb{T}^{2} \rightarrow$ $(-\infty, \infty)$ such that $f(x, y)$ is $2 \pi$-periodic with respect to each variable $x, y$ respectively. Let $\mathcal{T}_{m, \circ}$ (respectively $\mathcal{T}_{\circ, n}$ ) be the set of all trigonometric polynomial of degree at most $m$ (at most $n$ ) with respect to variable $x$ (variable $y$ ). We set $\mathcal{T}_{m, n}$ as the collection of all trigonometric polynomial of degree at most $m$ with respect to variable $x$ and of degree at most $n$ with respect to variable $y$. The best angular trigonometric approximation error is defined as

$$
Y_{m, n}(f)_{p, \omega}=\inf \left\{\|f-T-U\|_{p, \omega}: T \in \mathcal{T}_{m, o}, U \in \mathcal{T}_{\circ, n}\right\}
$$

where $1<p<\infty, \omega \in A_{p}$, and $f \in L_{\omega}^{p}$.

Using [15, Theorem 6$]$ we have

$$
\left\|f-C_{m, n}^{\alpha} f\right\|_{p, \omega} \rightarrow 0, \quad \text { as } m, n \rightarrow \infty,
$$

where $C_{m, n}^{\alpha} f$ is $\alpha$ th Cesàro mean of $f$. From this we can obtain that $C\left(\mathbb{T}^{2}\right)$, the class of continuous functions on $\mathbb{T}^{2}$, is a dense subset of $L_{\omega}^{p}$ for $1<p<\infty, \omega \in A_{p}$. Then

$$
Y_{m, n}(f)_{p, \omega} \leq C_{[\omega]_{A_{p}}, p, r}\left\|f-C_{m, n}^{\alpha} f\right\|_{p, \omega} \rightarrow 0
$$

and hence

$$
Y_{m, n}(f)_{p, \omega} \searrow 0, \quad \text { as } m, n \rightarrow \infty .
$$

This shows that approximation problems make sense in $L_{\omega}^{p}$ for $1<p<\infty, \omega \in A_{p}$.

Define Steklov type operators

$$
\begin{gathered}
\mathcal{S}_{\lambda, \tau ; \circ} f(x, y):=\lambda \int_{x+\tau-1 /(2 \lambda)}^{x+\tau+1 /(2 \lambda)} f(u, y) d u, \\
\mathcal{S}_{\circ ; \theta, \rho} f(x, y):=\theta \int_{y+\rho-1 /(2 \theta)}^{y+\rho+1 /(2 \theta)} f(x, v) d v, \\
\mathcal{S}_{\lambda, \tau ; \theta, \rho} f(x, y)=\mathcal{S}_{\lambda, \tau ; \circ}\left(\mathcal{S}_{\circ ; \theta, \rho} f(x, y)\right)=\lambda \theta \int_{x+\tau-1 /(2 \lambda)}^{x+\tau+1 /(2 \lambda)} \int_{y+\rho-1 /(2 \theta)}^{y+\rho+1 /(2 \theta)} f(u, v) d u d v .
\end{gathered}
$$

Theorem 2.1. We suppose that $1<p<\infty$ and $\omega \in A_{p}$.

(i) If $1 \leq \lambda<\infty$ and $|\tau| \leq \pi \lambda^{-1}$, then, the family of operators $\left\{\mathcal{S}_{\lambda, \tau ; \circ}\right\}_{1 \leq \lambda<\infty}$ is uniformly bounded (in $\lambda, \tau$ ) in $L_{\omega}^{p}$ :

$$
\left\|\mathcal{S}_{\lambda, \tau ; \circ} f\right\|_{p, \omega} \leq 108^{\frac{1}{p}} \pi^{\frac{2}{p}}[\omega]_{A_{p}}^{\frac{1}{p}}\|f\|_{p, \omega} .
$$

(ii) If $1 \leq \theta<\infty$ and $|\rho| \leq \pi \theta^{-1}$, then, the family of operators $\left\{\mathcal{S}_{\circ ; \theta, \rho}\right\}_{1 \leq \theta<\infty}$ is uniformly bounded (in $\theta, \rho)$ in $L_{\omega}^{p}$ :

$$
\left\|\mathcal{S}_{\circ ; \theta, \rho} f\right\|_{p, \omega} \leq 108^{\frac{1}{p}} \pi^{\frac{2}{p}}[\omega]_{A_{p}}^{\frac{1}{p}}\|f\|_{p, \omega} .
$$

(iii) If $1 \leq \lambda, \theta<\infty,|\tau| \leq \pi \lambda^{-1},|\rho| \leq \pi \theta^{-1}$, then, the family of operators $\left\{\mathcal{S}_{\lambda, \tau ; \theta, \rho}\right\}_{1 \leq \lambda, \theta<\infty}$ is uniformly bounded (in $\lambda, \tau$ and $\theta, \rho$ ) in $L_{\omega}^{p}$ :

$$
\left\|\mathcal{S}_{\lambda, \tau ; \theta, \rho} f\right\|_{p, \omega} \leq 108^{\frac{2}{p}} \pi^{\frac{4}{p}}[\omega]_{A_{p}}^{\frac{2}{p}}\|f\|_{p, \omega} .
$$

In this case Theorem 2.1 yields the following lemma.

Lemma 2.2 ([11, Theorem 3.3], [4]). If $1<p<\infty, \omega \in A_{p}$, and $f \in L_{\omega}^{p}$, then

$$
\left\{\left\|\sigma_{h, k} f\right\|_{p, \omega},\left\|\sigma_{h, \circ} f\right\|_{p, \omega},\left\|\sigma_{\circ, k} f\right\|_{p, \omega}\right\} \leq C_{[\omega]_{A_{p}}, p}\|f\|_{p, \omega},
$$

with constants depend only on $[\omega]_{A_{p}}$ and $p$. 


\subsection{Transference result}

At this stage we will need a transference result. If $f$ is $2 \pi$ periodic locally integrable function on $\mathbb{T}^{2}$, then (see Theorem 11.1 on page 211 of [9])

$$
\lim _{(h, k) \rightarrow(0,0)} \frac{1}{h k} \int_{x-h / 2}^{x+h / 2} \int_{y-k / 2}^{y+k / 2} f(t, \tau) d t d \tau=f(x, y)
$$

for almost every $(x, y) \in \mathbb{T}^{2}$. Then, for any $\zeta>0$ one can find $h_{0}, k_{0} \leq 1$ such that

$$
\mathcal{S}_{\frac{1}{h_{0}}, 0 ; \frac{1}{k_{0}}, 0} f(x, y)=\frac{1}{h_{0} k_{0}} \int_{x-h_{0} / 2}^{x+h_{0} / 2} \int_{y-k_{0} / 2}^{y+k_{0} / 2} f(t, \tau) d t d \tau>f(x, y)-\zeta
$$

almost every $(x, y) \in \mathbb{T}^{2}$. Throughout this work we will fix these $h_{0}, k_{0}$.

Let $1<p<\infty, \omega \in A_{p}, f \in L_{\omega}^{p}$,

$$
q:=\frac{p}{p-1},
$$

$$
G \in L_{\omega}^{q}\left(\mathbb{T}^{2}\right), \quad\|G\|_{q, \omega}=1
$$

and define, with $h_{0}, k_{0}$ of $(2.2)$,

$$
F_{f}(u, v):=\int_{\mathbb{T}^{2}} \mathcal{S}_{\frac{1}{h_{0}}, 0 ; \frac{1}{k_{0}}, 0} f(x+u, y+v)|G(x, y)| \omega(x, y) d x d y
$$

for $u, v \in \mathbb{T}$ satisfying $|u| \leq h_{0},|v| \leq k_{0}$.

Let $\tilde{F}_{f}(u, v)$ be a continuous function defined on $\mathbb{T}^{2}$ such that

i) $\tilde{F}_{f}(u, v)$ coincides with $F_{f}(u, v)$ on

$$
I_{h_{0}}^{k_{0}}:=\left\{(u, v) \in \mathbb{T}^{2}:|u| \leq h_{0},|v| \leq k_{0}\right\} .
$$

ii) $\max _{(u, v) \in \mathbb{T}^{2}}\left|\tilde{F}_{f}(u, v)\right| \leq \max _{(u, v) \in I_{h_{0}}^{k_{0}}}\left|F_{f}(u, v)\right|$.

Let $C\left(\mathbb{T}^{2}\right)$ denote the collection of continuous functions $f: \mathbb{T}^{2} \rightarrow \mathbb{R}$ with

$$
\|f\|_{C\left(\mathbb{T}^{2}\right)}:=\max \{|f(x, y)|: x, y \in \mathbb{T}\}<\infty .
$$

Lemma 2.3. If $1<p<\infty, \omega \in A_{p}$ and $f \in L_{\omega}^{p}$, then the function $F_{f}(u, v)$, defined above, is uniformly continuous on $I_{h_{0}}^{k_{0}}$.

Lemma 2.4. Let $1<p<\infty, q:=p /(p-1)$ and $\gamma$ be a weight on $\mathbb{T}^{2}$. Then

$$
\sup _{G \in L_{\omega}^{q}:\|G\|_{q, \omega}=1} \int_{\mathbb{T}^{2}} f(x, y) G(x, y) \omega(x, y) d x d y=\|f\|_{p, \omega}
$$

for $f \in L_{\omega}^{p}$.

Theorem 2.5. If $1<p<\infty, \omega \in A_{p}, f, g \in L_{\omega}^{p}$ and

$$
\left\|F_{g}\right\|_{C\left(I_{h_{0}}^{k_{0}}\right)} \leq c\left\|F_{f}\right\|_{C}\left(I_{h_{0}}^{k_{0}}\right),
$$

then

$$
\|g\|_{p, \omega} \leq c 108^{\frac{2}{p}} \pi^{\frac{4}{p}}[\omega]_{A_{p}}^{\frac{2}{p}}\|f\|_{p, \omega} .
$$




\subsection{Fractional order modulus of smoothness}

Now, we consider the fractional smoothness $\Omega_{r}(\cdot, \delta, \xi)_{p, \omega}, r>0$, suitable for some weighted spaces. Note that classical non-weighted fractional smoothness $\omega_{r}(f, \cdot)_{p}, r>0$, was defined by P. L. Butzer, H. Dyckhoff, E. Görlich, R. L. Stens [7], and R. Taberski [28] and may be some others. See also [27].

Firstly we discuss boundedness of Steklov operators.

Remark 2.6. (i) If $\mathcal{F} \in C\left(\mathbb{T}^{2}\right)$ then we know that

$$
\left\|\sigma_{h, \circ} \mathcal{F}\right\|_{C\left(\mathbb{T}^{2}\right)} \leq\|\mathcal{F}\|_{C\left(\mathbb{T}^{2}\right)}, \quad\left\|\sigma_{\circ, k} \mathcal{F}\right\|_{C\left(\mathbb{T}^{2}\right)} \leq\|\mathcal{F}\|_{C\left(\mathbb{T}^{2}\right)}, \quad\left\|\sigma_{h, k} \mathcal{F}\right\|_{C\left(\mathbb{T}^{2}\right)} \leq\|\mathcal{F}\|_{C\left(\mathbb{T}^{2}\right)} .
$$

Hence

and these give that

$$
\left\|\nabla_{h, \circ}^{r, \circ} \mathcal{F}\right\|_{C\left(\mathbb{T}^{2}\right)} \leq 2^{r}\|\mathcal{F}\|_{C\left(\mathbb{T}^{2}\right)}, \quad\left\|\nabla_{\circ, k}^{\circ, r} \mathcal{F}\right\|_{C\left(\mathbb{T}^{2}\right)} \leq 2^{r}\|\mathcal{F}\|_{C\left(\mathbb{T}^{2}\right)}
$$

for $\mathcal{F} \in C\left(\mathbb{T}^{2}\right)$.

$$
\left\|\nabla_{h, k}^{r, r} \mathcal{F}\right\|_{C\left(\mathbb{T}^{2}\right)} \leq 2^{2 r}\|\mathcal{F}\|_{C\left(\mathbb{T}^{2}\right)}
$$

(ii) Using (i)

$$
\begin{gathered}
\left\|F_{\nabla_{h, \circ}^{r, \circ} \mathcal{F}}\right\| \|_{C\left(I_{h_{0}}^{k_{0}}\right)}=\max _{u, v \in I_{h_{0}}^{k_{0}}}\left|\int_{\mathbb{T}^{2}} \nabla_{h, \circ}^{r, \circ} \mathcal{S}_{\frac{1}{h_{0}}, 0 ; \frac{1}{k_{0}}, 0} \mathcal{F}(x+u, y+v)\right| G(x, y)|\omega(x, y) d x d y| \\
=\max _{u, v \in I_{h_{0}}^{k_{0}}}\left|\nabla_{h, \circ}^{r, \circ} \int_{\mathbb{T}^{2}} \mathcal{S}_{\frac{1}{h_{0}}, 0 ; \frac{1}{k_{0}}, 0} \mathcal{F}(x+u, y+v)\right| G(x, y)|\omega(x, y) d x d y|=\max _{u, v \in I_{h_{0}}^{k_{0}}}\left|\nabla_{h, \circ}^{r, \circ} F_{\mathcal{F}}\right| \\
\leq 2^{r} \max _{u, v \in I_{h_{0}}^{k_{0}}}\left|F_{\mathcal{F}}\right|=2^{r}\left\|F_{\mathcal{F}}\right\|_{C}\left(I_{h_{0}}^{k_{0}}\right) .
\end{gathered}
$$

The same method gives that

$$
\left\|F_{\nabla_{\circ, k}^{\circ, r} \mathcal{F}}\right\|_{C\left(I_{h_{0}}^{k_{0}}\right)} \leq 2^{r}\left\|F_{\mathcal{F}}\right\|_{C}\left(I_{h_{0}}^{k_{0}}\right) .
$$

Using the last two inequalities we find

$$
\left\|F_{\nabla_{h, k}^{r, r} \mathcal{F}}\right\|_{C\left(I_{h_{0}}^{k_{0}}\right)} \leq 2^{2 r}\left\|F_{\mathcal{F}}\right\|_{C\left(I_{h_{0}}^{k_{0}}\right)} .
$$

Using Theorem 2.5 we find

$$
\left\|\nabla_{h, \circ}^{r, \circ} f\right\|_{p, \omega} \leq 108^{\frac{2}{p}} \pi^{\frac{4}{p}}[\omega]_{A_{p}}^{\frac{2}{p}} 2^{r}\|f\|_{p, \omega}, \quad\left\|\nabla_{\circ, k}^{\circ, r} f\right\|_{p, \omega} \leq 108^{\frac{2}{p}} \pi^{\frac{4}{p}}[\omega]_{A_{p}}^{\frac{2}{p}} 2^{r}\|f\|_{p, \omega}
$$

and, therefore

for $f \in L_{\omega}^{p}$.

$$
\left\|\nabla_{h, k}^{r, r} f\right\|_{p, \omega} \leq 2^{2 r} 108^{\frac{4}{p}} \pi^{\frac{8}{p}}[\omega]_{A_{p}}^{\frac{4}{p}}\|f\|_{p, \omega}
$$

The last remark implies the following.

Corollary 2.7. Let $p \in(1, \infty), \omega \in A_{p}, r \in \mathbb{R}^{+}$and $f \in L_{\omega}^{p}$. Then

(i) There exists a constant $C_{p, \omega, r}>0$, independent of $h, k$, such that

$$
\left\{\left\|\nabla_{h, \circ}^{r, o} f\right\|_{p, \omega},\left\|\nabla_{\circ, k}^{\circ, r} f\right\|_{p, \omega},\left\|\nabla_{h, k}^{r, r} f\right\|_{p, \omega}\right\} \leq C_{p, \omega, r}\|f\|_{p, \omega}
$$

holds for $r>0$.

(ii) There holds

$$
\Omega_{r}\left(f, \delta_{1}, \delta_{2}\right)_{p, \omega} \leq C_{[\omega]_{A_{p}}, p, r}\|f\|_{p, \omega}
$$

with constant depending only on $[\omega]_{A_{p}}$ and $p, r$. 
(iii) $\Omega_{r}(f, 0,0)_{p, \omega}=0$.

(iv) $\Omega_{r}\left(f, \delta_{1}, \delta_{2}\right)_{p, \omega}$ is subadditive with respect to $f$.

(v) $\Omega_{r}\left(f, \delta_{1}, \delta_{2}\right)_{p, \omega} \leq \Omega_{r}\left(f, t_{1}, t_{2}\right)_{p, \omega}$ for $0 \leq \delta_{i} \leq t_{i} ; \quad i=1,2$.

\subsection{Some means of Fourier series}

Let $1<p<\infty, \omega \in A_{p}$, and $f \in L_{\omega}^{p}$, then one can find a $p^{*} \in(1, \infty)$ with $f \in L^{p^{*}}\left(\mathbb{T}^{2}\right)$. Hence,

$$
L_{\omega}^{p} \subset L^{p^{*}}
$$

Let $1<p<\infty, \omega \in A_{p}$ and

$$
\sum_{n_{1}=0}^{\infty} \sum_{n_{2}=0}^{\infty} A_{n_{1}, n_{2}}(x, y)
$$

be the corresponding Fourier series for $f \in L_{\omega}^{p} \subset L^{1}$. For the Fourier series (2.8) of $f \in L_{\omega}^{p}$, $1<p<\infty, \omega \in A_{p}$ we define

$$
\begin{aligned}
& S_{m, \circ}(f)(x, y)=\sum_{n_{1}=0}^{m} \sum_{n_{2}=0}^{\infty} A_{n_{1}, n_{2}}(x, y, f), S_{\circ, n}(f)(x, y)=\sum_{n_{1}=0}^{\infty} \sum_{n_{2}=0}^{n} A_{n_{1}, n_{2}}(x, y, f), \\
& S_{m, n}(f)(x, y)=S_{m, \circ}\left(S_{\circ, n}(f)\right)(x, y)=\sum_{n_{1}=0}^{m} \sum_{n_{2}=0}^{n} A_{n_{1}, n_{2}}(x, y, f),
\end{aligned}
$$

and de la Valleè Poussin means of $f$

$$
\begin{aligned}
& V_{m, \circ}(f)=\frac{1}{m+1} \sum_{k=m}^{2 m-1} S_{k, \circ}(f), V_{\circ, n}(f)=\frac{1}{n+1} \sum_{l=n}^{2 n-1} S_{\circ, l}(f), \\
& V_{m, n}(f)=V_{m, \circ}\left(V_{\circ, n}(f)\right)=\frac{1}{(n+1)(m+1)} \sum_{k=m}^{2 m-1} \sum_{l=n}^{2 n-1} S_{k, l}(f) .
\end{aligned}
$$

In what follows, $A \lesssim B$ will mean that the inequality $A \leq C B$ holds. If $A \lesssim B$ and $B \lesssim A$ we will write $A \approx B$.

Lemma 2.8 ([4]). If $1<p<\infty, \omega \in A_{p}, f \in L_{\omega}^{p}$, then

$$
\left\{\left\|S_{m, \circ}(f)\right\|_{p, \omega},\left\|S_{\circ, n}(f)\right\|_{p, \omega},\left\|S_{m, n}(f)\right\|_{p, \omega}\right\} \lesssim\|f\|_{p, \omega}
$$

$$
\left\{\left\|V_{m, \circ}(f)\right\|_{p, \omega},\left\|V_{\circ, n}(f)\right\|_{p, \omega},\left\|V_{m, n}(f)\right\|_{p, \omega}\right\} \lesssim\|f\|_{p, \omega}
$$

$$
\left\|f-W_{m, n} f\right\|_{p, \omega} \lesssim Y_{m, n}(f)_{p, \omega}
$$

where

$$
W_{m, n} f(x, y):=\left(V_{m, \circ}(f)+V_{\circ, n}(f)-V_{m, n}(f)\right)(x, y)
$$

and all constants depending only on $[\omega]_{A_{p}}$ and $p$. 


\subsection{Bernstein inequalities}

Lemma 2.9 (Bernstein's Inequality, [4]). If $1<p<\infty, \omega \in A_{p}, T_{1} \in \mathcal{T}_{m, \circ}, T_{2} \in \mathcal{T}_{\circ, n}, T_{3} \in$ $\mathcal{T}_{m, n}, j, l \in \mathbb{N}$, then

$$
\left\|T_{1}^{(j, \circ)}\right\|_{p, \omega} \lesssim m^{j}\left\|T_{1}\right\|_{p, \omega}, \quad\left\|T_{2}^{(\circ, l)}\right\|_{p, \omega} \lesssim n^{l}\left\|T_{2}\right\|_{p, \omega},
$$

and, as a result,

$$
\left\|T_{3}^{(j, l)}\right\|_{p, \omega} \lesssim m^{j} n^{l}\left\|T_{3}\right\|_{p, \omega}
$$

with constants depending only on $[\omega]_{A_{p}}$ and $p$.

Suppose that $\|\cdot\|_{L_{\omega}^{p}}$ is the one dimensional norm in $L_{\omega}^{p}(\mathbb{T})$,

$$
\sigma_{h} f(x):=\frac{1}{2 h} \int_{x-h}^{x+h} f(t) d t
$$

and $\mathfrak{U}_{n}$ is the collection of all one dimensional trigonometric polynomial of degree at most $n$.

Lemma $2.10([3])$. Let $r \in \mathbb{R}^{+}, n \in \mathbb{N}, p \in(1, \infty), \omega \in A_{p}$ and $U_{n} \in \mathfrak{U}_{n}$. Then

$$
h^{2 r}\left\|U_{n}^{(2 r)}\right\|_{L_{\omega}^{p}} \lesssim\left\|\left(I-\sigma_{h}\right)^{r} U_{n}\right\|_{L_{\omega}^{p}}
$$

holds for any $h \in(0, \pi / n]$ with some constant depending only on $r, p$ and $[\omega]_{A_{p}}$.

From the last lemma we obtain that if $T_{m} \in \mathfrak{U}_{m}$ then

$$
\begin{aligned}
\left(\int_{\mathbb{T}}\left|T_{m}^{(r)}(x)\right|^{p} \omega(x) d x\right)^{1 / p} & \lesssim m^{r}\left(\int_{\mathbb{T}}\left|\left[T_{m}(x)-\sigma_{\frac{1}{m}} T_{m}(x)\right]^{r / 2}\right|^{p} \omega(x) d x\right)^{1 / p} \\
& \lesssim m^{r}\left(\int_{\mathbb{T}}\left|T_{m}(x)\right|^{p} \omega(x) d x\right)^{1 / p}
\end{aligned}
$$

and, accordingly

$$
\int_{\mathbb{T}}\left|T_{m}^{(r)}(x)\right|^{p} \omega(x) d x \lesssim m^{r p} \int_{\mathbb{T}}\left|T_{m}(x)\right|^{p} \omega(x) d x .
$$

Lemma 2.11 (Fractional Bernstein Inequality). Let $1<p<\infty, \omega \in A_{p}, T_{1} \in \mathcal{T}_{m, \circ}, T_{2} \in$ $\mathcal{T}_{\circ, n}, T_{3} \in \mathcal{T}_{m, n}, j, l \in \mathbb{R}^{+}$. Then

$$
\left\|T_{1}^{(j, \circ)}\right\|_{p, \omega} \lesssim m^{j}\left\|T_{1}\right\|_{p, \omega}, \quad\left\|T_{2}^{(\circ, l)}\right\|_{p, \omega} \lesssim n^{l}\left\|T_{2}\right\|_{p, \omega},
$$

and, as a result,

$$
\left\|T_{3}^{(j, l)}\right\|_{p, \omega} \lesssim m^{j} n^{l}\left\|T_{3}\right\|_{p, \omega}
$$

with constants depending only on $[\omega]_{A_{p}}$ and $p$.

As a corollary of Lemma 2.11 and [4] we have the following lemma.

Lemma 2.12. For $1<p<\infty, \omega \in A_{p}, f \in L_{\omega}^{p}, \varsigma, l \in \mathbb{R}^{+}$there exists a constant depending only on $[\omega]_{A_{p}}$ and $p$ so that

$$
\begin{gathered}
\left\|\left[\varphi_{i, j}(f)\right]^{(\varsigma, l)}\right\|_{p, \omega} \lesssim 2^{i \varsigma} 2^{j l} Y_{\left\lfloor 2^{i-1}\right\rfloor,\left\lfloor 2^{j-1}\right\rfloor}(f)_{p, \omega} \\
\left\|\left[\psi_{i, j}(f)\right]^{(\varsigma, \circ)}\right\|_{p, \omega} \lesssim 2^{i \varsigma} Y_{\left\lfloor 2^{i-1}\right\rfloor, 2^{j}}(f)_{p, \omega}, \quad\left\|\left[h_{i, j}(f)\right]^{(\circ, l)}\right\|_{p, \omega} \lesssim 2^{j l} Y_{2^{i},\left\lfloor 2^{j-1}\right\rfloor}(f)_{p, \omega},
\end{gathered}
$$


where

$$
\begin{gathered}
V_{2^{i}, 2^{j}}(f)-V_{2^{i},\left\lfloor 2^{j-1}\right\rfloor}(f)-V_{\left\lfloor 2^{i-1}\right\rfloor, 2^{j}}(f)+V_{\left\lfloor 2^{i-1}\right\rfloor,\left\lfloor 2^{j-1}\right\rfloor}(f)=: \varphi_{i, j}(f) \in \mathcal{T}_{2^{i+1}-1,2^{j+1}-1}, \\
V_{2^{i}, \circ}\left(f-V_{\circ, 2^{j}}(f)\right)-V_{\left\lfloor 2^{i-1}\right\rfloor, \circ}\left(f-V_{\circ, 2^{j}}(f)\right)=: \psi_{i, j}(f) \in \mathcal{T}_{2^{i+1}-1, \circ}, \\
V_{\circ, 2^{j}}\left(f-V_{2^{i}, \circ}(f)\right)-V_{\circ,\left\lfloor 2^{j-1}\right\rfloor}\left(f-V_{2^{i}, \circ}(f)\right)=: h_{i, j}(f) \in \mathcal{T}_{\circ, 2^{j+1}-1} .
\end{gathered}
$$

\section{Favard inequalities}

Lemma 3.1. Let $1<p<\infty, \omega \in A_{p}$, and $r \in \mathbb{R}^{+}$. Then, there exist constants depending only on $[\omega]_{A_{p}}$ and $p, r$ such that

$$
\begin{gathered}
Y_{m, n}\left(g_{1}\right)_{p, \omega} \lesssim(m+1)^{-2 r}\left\|g_{1}^{(2 r, \circ)}\right\|_{p, \omega}, \quad g_{1} \in W_{p, \omega}^{2 r, \circ}, \\
Y_{m, n}\left(g_{2}\right)_{p, \omega} \lesssim(n+1)^{-2 r}\left\|g_{2}^{(\circ, 2 r)}\right\|_{p, \omega}, \quad g_{2} \in W_{p, \omega}^{\circ, 2 r}, \\
Y_{m, n}(g)_{p, \omega} \lesssim(m+1)^{-2 r}(n+1)^{-2 r}\left\|g^{(2 r, 2 r)}\right\|_{p, \omega}, \quad g \in W_{p, \omega}^{2 r, 2 r} .
\end{gathered}
$$

Theorem $3.2([5])$. Let $1<p<\infty, \omega \in A_{p}, f \in L_{\omega}^{p}$, and $r \in \mathbb{N}$. Then there exist constants depending only on $[\omega]_{A_{p}}$ and $p, r$ so that

$$
\begin{aligned}
\Omega_{r}\left(g_{1}, \delta, \cdot\right)_{p, \omega} & \lesssim \delta^{2} \Omega_{r-1}\left(g_{1}^{(2, \circ)}, \delta, \cdot\right)_{p, \omega}, \quad g_{1} \in W_{p, \omega}^{2, \circ}, \\
\Omega_{r}\left(g_{2}, \cdot, \xi\right)_{p, \omega} & \lesssim \xi^{2} \Omega_{r-1}\left(g_{2}^{(\circ, 2)}, \cdot, \xi\right)_{p, \omega}, \quad g_{2} \in W_{p, \omega}^{\circ, 2} \\
\Omega_{r}(g, \delta, \xi)_{p, \omega} & \lesssim \delta^{2} \xi^{2} \Omega_{r-1}\left(g^{(2,2)}, \delta, \xi\right)_{p, \omega}, \quad g \in W_{p, \omega}^{2,2},
\end{aligned}
$$

hold for $\delta, \xi>0$.

Corollary 3.3. Let $1<p<\infty, m, n \in \mathbb{N}, \omega \in A_{p}$, and $r \in \mathbb{R}^{+}$. Then there exist constants depending only on $[\omega]_{A_{p}}$ and $p, r$ such that

$$
\begin{aligned}
\Omega_{r}\left(T_{1}, \pi /(m+1), \cdot\right)_{p, \omega} & \lesssim(m+1)^{-2 r}\left\|T_{1}^{(2 r, \circ)}\right\|_{p, \omega}, \quad T_{1} \in \mathcal{T}_{m, \circ}, \\
\Omega_{r}\left(T_{2}, \cdot, \pi /(n+1)\right)_{p, \omega} & \lesssim(n+1)^{-2 r}\left\|T_{2}^{(\circ, 2 r)}\right\|_{p, \omega}, \quad T_{2} \in \mathcal{T}_{\circ, n}, \text { and } \\
\Omega_{r}\left(T_{3}, \pi /(m+1), \pi /(n+1)\right)_{p, \omega} & \lesssim((m+1)(n+1))^{-2 r}\left\|T_{3}^{(2 r, 2 r)}\right\|_{p, \omega}, \quad T_{3} \in \mathcal{T}_{m, n}
\end{aligned}
$$

hold.

For $r=1$ Corollary 3.3 was proved in [4].

\section{Proof of the results}

Proof of Theorem 2.1. From [6, Th.10] we have, for almost every $y$,

$$
\int_{\mathbb{T}}\left|\mathcal{S}_{\lambda, \tau ; \circ} f(x, y)\right|^{p} \omega(x, y) d x \leq 108 \pi^{2}[\omega]_{A_{p}} \int_{\mathbb{T}}|f(x, y)|^{p} \omega(x, y) d x .
$$

The last inequality imply that

$$
\begin{gathered}
\int_{\mathbb{T}^{2}}\left|\mathcal{S}_{\lambda, \tau ; \circ} f(x, y)\right|^{p} \omega(x, y) d x d y \leq 108 \pi^{2}[\omega]_{A_{p}} \int_{\mathbb{T}^{2}}|f(x, y)|^{p} \omega(x, y) d x d y, \\
\left\|\mathcal{S}_{\lambda, \tau ; \circ} f\right\|_{p, \omega} \leq 108^{\frac{1}{p}} \pi^{\frac{2}{p}}[\omega]_{A_{p}}^{\frac{1}{p}}\|f\|_{p, \omega} .
\end{gathered}
$$

Completely similar arguments give

$$
\left\|\mathcal{S}_{\circ ; \theta, \rho} f\right\|_{p, \omega} \leq 108^{\frac{1}{p}} \pi^{\frac{2}{p}}[\omega]_{A_{p}}^{\frac{1}{p}}\|f\|_{p, \omega} .
$$


Summing up obtained inequalities

as desired.

$$
\begin{gathered}
\left\|\mathcal{S}_{\lambda, \tau ; \theta, \rho} f\right\|_{p, \omega}=\left\|\mathcal{S}_{\lambda, \tau ; \circ}\left(\mathcal{S}_{\circ ; \theta, \rho} f\right)\right\|_{p, \omega} \\
\leq 108^{\frac{1}{p}} \pi^{\frac{2}{p}}[\omega]_{A_{p}}^{\frac{1}{p}}\left\|\mathcal{S}_{\circ ; \theta, \rho} f\right\|_{p, \omega} \leq 108^{\frac{2}{p}} \pi^{\frac{4}{p}}[\omega]_{A_{p}}^{\frac{2}{p}}\|f\|_{p, \omega}
\end{gathered}
$$

Proof of Lemma 2.3. Since $C\left[\mathbb{T}^{2}\right]$ is a dense subset of $f \in L_{\omega}^{p}, 1<p<\infty, \omega \in A_{p}$, we consider only the case $f \in C\left[\mathbb{T}^{2}\right]$. Let $0<h, k \leq 1$ be given. Suppose that $u, v \in I_{h_{0}}^{k_{0}}$ and $\varepsilon>0$. When $\max \left\{\left|u_{1}\right|,\left|v_{1}\right|\right\} \rightarrow 0$ we have $x+u+u_{1} \rightarrow x+u$ and $y+v+v_{1} \rightarrow y+v$. Also

$$
\sigma_{h, k} f\left(x+u+u_{1}, y+v+v_{1}\right) \rightarrow \sigma_{h, k} f(x+u, y+v) .
$$

Then one can find a $\delta(\varepsilon)>0$ so that

$$
\left|\sigma_{h, k} f\left(x+u+u_{1}, y+v+v_{1}\right)-\sigma_{h, k} f(x+u, y+v)\right|<\varepsilon
$$

for $\left|u_{1}\right|,\left|v_{1}\right|<\delta$. Hence,

$$
\begin{gathered}
\left|F_{f}\left(u+u_{1}, v+v_{1}\right)-F_{f}(u, v)\right| \\
=\left|\int_{\mathbb{T}^{2}}\left[\sigma_{h, k} f\left(x+u+u_{1}, y+v+v_{1}\right)-\sigma_{h, k} f(x+u, y+v)\right]\right| G(x, y)|\omega(x, y) d x d y| \\
\leq \varepsilon \int_{\mathbb{T}^{2}}|G(x, y)| \omega(x, y) d x d y \leq \varepsilon\|\omega\|_{1,1}^{1 / p}\|G\|_{q, \omega}=\varepsilon\|\omega\|_{1,1}^{1 / p}
\end{gathered}
$$

for $\left|u_{1}\right|,\left|v_{1}\right|<\delta$. A density result now give that $F_{f}(u, v)$ is uniformly continuous on $I_{h_{0}}^{k_{0}}$. From this we can write

$$
\tilde{F}_{f}(u, v) \in C\left(\mathbb{T}^{2}\right) .
$$

Proof of Lemma 2.4. (2.4) is known (see e.g., Proposition 3.1 of [9, p.250] for any measure $d \mu$ :

$$
\sup _{G \in L_{q, d \mu}:\|G\|_{q, d \mu}=1} \int_{\mathbb{T}^{2}} f(x, y) G(x, y) d \mu=\|f\|_{p, d \mu}
$$

When $d \mu=\omega(x, y) d x d y(2.4)$ also holds.

Proof of Theorem 2.5. Let $0<h, k \leq 1, \omega \in A_{p}, 1<p<\infty$ and $f, g \in L_{\omega}^{p}$. In this case

$$
\begin{aligned}
\left\|F_{g}\right\|_{C\left(I_{h_{0}} k_{0}\right.} & \leq c\left\|F_{f}\right\|_{C}\left(I_{h_{0}}^{k_{0}}\right) \\
& =c\left\|\int_{\mathbb{T}^{2}} \mathcal{S}_{\frac{1}{h_{0}}, 0 ; \frac{1}{k_{0}}, 0} f(x+u, y+v)|G(x, y)| \omega(x, y) d x d y\right\|_{C\left(I_{h_{0}}^{k_{0}}\right)} \\
& =c\left|\max _{u, v \in I_{h_{0}}^{k_{0}}} \int \mathbb{T}^{2} \mathcal{S}_{\frac{1}{h_{0}}, 0 ; \frac{1}{k_{0}}, 0} f(x+u, y+v)\right| G(x, y)|\omega(x, y) d x d y| \\
& \leq c \max _{u, v \in I_{h_{0}}^{k_{0}}}\left\|\mathcal{S}_{\frac{1}{h_{0}}, 0 ; \frac{1}{k_{0}}, 0} f(\cdot+u, \cdot+v)\right\|_{p, \omega}\|G\|_{q, \omega} \\
& \leq c \max _{u, v \in I_{h_{0}}^{k_{0}}}\left\|\mathcal{S}_{\frac{1}{h_{0}}, u ; \frac{1}{k_{0}}, v}\right\|_{L_{\omega}^{p} \rightarrow L_{\omega}^{p}}\|f\|_{p, \omega}, \quad \text { (by Theorem 2.1) } \\
& \leq c 108^{\frac{2}{p}} \pi^{\frac{4}{p}}[\omega]_{A_{p}}^{\frac{2}{p}}\|f\|_{p, \omega} .
\end{aligned}
$$


On the other hand, for any $\varepsilon>0$ and appropriately chosen $G \in L_{\omega}^{q}\left(\mathbb{T}^{2}\right)$ with $\langle g, G\rangle=$ $\int_{\mathbb{T}^{2}} g(x, y) G(x, y) \omega(x, y) d x d y \geq\|g\|_{p, \omega}-\varepsilon,\|G\|_{q, \omega}=1$, (see Lemma 2.4 above), one can find

$$
\begin{aligned}
\left\|F_{g}\right\|_{C\left(I_{h_{0}}\right)} & \geq\left|F_{g}(0,0)\right| \geq \int_{\mathbb{T}^{2}} \mathcal{S}_{\frac{1}{h_{0}}, 0 ; \frac{1}{k_{0}}, 0} g(x, y)|G(x, y)| \omega(x, y) d x d y \\
& \geq \int_{\mathbb{T}^{2}} g(x, y)|G(x, y)| \omega(x, y) d x d y-\zeta \int_{\mathbb{T}^{2}}|G(x, y)| \omega(x, y) d x d y \\
& \geq\|g\|_{p, \omega}-\varepsilon-\zeta\|\omega\|_{1,1}^{1 / p} .
\end{aligned}
$$

Since $\varepsilon, \zeta>0$ is arbitrary, from the last inequality, we have

$$
\left\|F_{g}\right\|_{C\left(I_{h_{0}}^{k_{0}}\right)} \geq\|g\|_{p, \omega}
$$

and hence

$$
\|g\|_{p, \omega} \leq\left\|F_{g}\right\|_{C\left(I_{h_{0}}^{k_{0}}\right)} \leq c\left\|F_{f}\right\|_{C\left(I_{h_{0}}^{k_{0}}\right)} \leq c 108^{\frac{2}{p}} \pi^{\frac{4}{p}}[\omega]_{A_{p}}^{\frac{2}{p}}\|f\|_{p, \omega} .
$$

This gives required result.

Proof of Theorem 2.7. Inequalities (2.7) hold with (2.5)-(2.6).

Proof of Lemma 2.11. From (2.11), we have for almost every $y$

$$
\int_{\mathbb{T}}\left|T_{1}^{(j, \circ)}(x, y)\right|^{p} \omega(x, y) d x \lesssim m^{j p} \int_{\mathbb{T}}\left|T_{1}(x, y)\right|^{p} \omega(x, y) d x .
$$

Hence

$$
\begin{aligned}
& \int_{\mathbb{T}^{2}}\left|T_{1}^{(j, \circ)}(x, y)\right|^{p} \omega(x, y) d x d y \lesssim m^{j p} \int_{\mathbb{T}^{2}}\left|T_{1}(x, y)\right|^{p} \omega(x, y) d x d y \\
&\left\|T_{1}^{(j, \circ)}\right\|_{p, \omega} \lesssim m^{j}\left\|T_{1}\right\|_{p, \omega} .
\end{aligned}
$$

From (2.11), we have for almost every $x$

$$
\int_{\mathbb{T}}\left|T_{2}^{(\circ, l)}(x, y)\right|^{p} \omega(x, y) d y \lesssim n^{l p} \int_{\mathbb{T}}\left|T_{2}(x, y)\right|^{p} \omega(x, y) d y .
$$

Then

holds.

$$
\begin{aligned}
& \int_{\mathbb{T}^{2}}\left|T_{2}^{(\circ, l)}(x, y)\right|^{p} \omega(x, y) d x d y \lesssim n^{l p} \int_{\mathbb{T}^{2}}\left|T_{2}(x, y)\right|^{p} \omega(x, y) d x d y, \\
& \left\|T_{2}^{(\circ, l)}\right\|_{p, \omega} \lesssim n^{l}\left\|T_{2}\right\|_{p, \omega}
\end{aligned}
$$

Proof of Lemma 3.1. We consider (3.1). We have

$$
\begin{gathered}
\left\|g_{1}-S_{m, \circ}\left(g_{1}\right)-S_{\circ, n}\left(g_{1}\right)+S_{m, n}\left(g_{1}\right)\right\|_{p, \omega}=\left\|\sum_{i=m+1}^{\infty} \sum_{j=n+1}^{\infty} A_{i, j}\left(x, y, g_{1}\right)\right\|_{p, \omega} \\
=\left\|\sum_{i=m+1}^{\infty} \sum_{j=n+1}^{\infty} \frac{1}{i^{2 r}} i^{2 r} A_{i, j}\left(x+\frac{\pi}{2}, y, g_{1}\right) \cos \pi\right\|_{p, \omega} \\
=\left\|-\sum_{i=m+1}^{\infty} \sum_{j=n+1}^{\infty} \frac{1}{i^{2 r}} A_{i, j}\left(x, y, g_{1}^{(2 r, \circ)}\right)\right\|_{p, \omega}
\end{gathered}
$$




$$
\begin{gathered}
=\left\|\sum_{i=m+1}^{\infty} \sum_{j=n+1}^{\infty} \frac{\left[S_{i, j}\left[g_{1}^{(2 r, o)}\right]-S_{i, j-1}\left[g_{1}^{(2 r, \circ)}\right]-S_{i-1, j}\left[g_{1}^{(2 r, \circ)}\right]+S_{i-1, j-1}\left[g_{1}^{(2 r, \circ)}\right]\right]}{i^{2 r}}\right\|_{p, \omega} \\
=\left\|\sum_{i=m+1}^{\infty}\left[\frac{1}{(i+1)^{2 r}}-\frac{1}{i^{2 r}}\right] S_{i, m}\left(g_{1}^{(2 r, \circ)}\right)+\frac{1}{(m+1)^{2}} S_{m, n}\left(g_{1}^{(2 r, \circ)}\right)\right\|_{p, \omega} \\
\leq \sum_{i=m+1}^{\infty}\left|\frac{1}{(i+1)^{2 r}}-\frac{1}{i^{2 r}}\right|\left\|S_{i, m}\left(g_{1}^{(2 r, \circ)}\right)\right\|_{p, \omega}+\frac{1}{(m+1)^{2 r}}\left\|S_{m, n}\left(g_{1}^{(2 r, \circ)}\right)\right\|_{p, \omega} \\
\leq\left\|g_{1}^{(2 r, \circ)}\right\|_{p, \omega}\left(\sum_{i=m+1}^{\infty}\left(\frac{1}{i^{2 r}}-\frac{1}{(i+1)^{2 r}}\right)+\frac{1}{(m+1)^{2 r}}\right) \leq \frac{C}{(m+1)^{2 r}}\left\|g_{1}^{(2 r, \circ)}\right\|_{p, \omega} .
\end{gathered}
$$

Using

$$
\begin{gathered}
Y_{m, n}\left(g_{1}\right)_{p, \omega}=Y_{m, n}\left(g_{1}-S_{m, \circ}\left(g_{1}\right)-S_{\circ, n}\left(g_{1}\right)+S_{m, n}\left(g_{1}\right)\right)_{p, \omega} \\
\leq\left\|g_{1}-S_{m, \circ}\left(g_{1}\right)-S_{\circ, n}\left(g_{1}\right)+S_{m, n}\left(g_{1}\right)\right\|_{p, \omega}
\end{gathered}
$$

one can find (3.1). The same method give

$$
\left\|g_{2}-S_{\circ, n}\left(g_{2}\right)-S_{m, \circ}\left(g_{2}\right)+S_{m, n}\left(g_{2}\right)\right\|_{p, \omega} \lesssim \frac{1}{(n+1)^{2 r}}\left\|g_{2}^{(\circ, 2 r)}\right\|_{p, \omega}
$$

and

$$
\begin{gathered}
Y_{m, n}\left(g_{2}\right)_{p, \omega}=Y_{m, n}\left(g_{2}-S_{\circ, n}\left(g_{2}\right)-S_{m, \circ}\left(g_{2}\right)+S_{m, n}\left(g_{2}\right)\right)_{p, \omega} \\
\leq\left\|g_{1}-S_{\circ, n}\left(g_{1}\right)-S_{m, \circ}\left(g_{1}\right)+S_{m, n}\left(g_{1}\right)\right\|_{p, \omega} \lesssim \frac{1}{(n+1)^{2 r}}\left\|g_{2}^{(\circ, 2 r)}\right\|_{p, \omega} .
\end{gathered}
$$

Considering (3.2), we find

$$
\begin{aligned}
& \left\|g-S_{m, \circ}(g)-S_{\circ, n}(g)+S_{m, n}(g)\right\|_{p, \omega} \\
& =\left\|\sum_{i=m+1}^{\infty} \sum_{j=n+1}^{\infty} A_{i, j}(x, y, g)\right\|_{p, \omega}=\left\|\sum_{i=m+1}^{\infty} \sum_{j=n+1}^{\infty} \frac{i^{2 r} j^{2 r}}{i^{2 r} j^{2 r}} A_{i, j}\left(x+\frac{\pi}{2}, y+\frac{\pi}{2}, g\right) \cos ^{2} \pi\right\|_{p, \omega} \\
& =\left\|\sum_{i=m+1}^{\infty} \sum_{j=n+1}^{\infty} \frac{1}{i^{2 r} j^{2 r}} A_{i, j}\left(x, y, g^{(2 r, 2 r)}\right)\right\|_{p, \omega}=\left\|\sum_{i=m+1}^{\infty} \sum_{j=n+1}^{\infty} \frac{1}{i^{2 r} j^{2 r}} A_{i, j}(x, y, \Upsilon)\right\|_{p, \omega} \\
& =\left\|\sum_{i=m+1}^{\infty} \sum_{j=n+1}^{\infty} \frac{1}{i^{2 r} j^{2 r}}\left(S_{i, j}(\Upsilon)-S_{i, j-1}(\Upsilon)-S_{i-1, j}(\Upsilon)+S_{i-1, j-1}(\Upsilon)\right)\right\|_{p, \omega} \\
& =\| \sum_{i=m+1}^{\infty} \sum_{j=n+1}^{\infty}\left[\frac{1}{(i+1)^{2 r}}-\frac{1}{i^{2 r}}\right]\left[\frac{1}{(j+1)^{2 r}}-\frac{1}{j^{2 r}}\right] S_{i, j}(\Upsilon)+ \\
& +\frac{1}{(m+1)^{2 r}} \sum_{j=n+1}^{\infty}\left[\frac{1}{(j+1)^{2 r}}-\frac{1}{j^{2 r}}\right] S_{m, j}(\Upsilon)+\frac{1}{(n+1)^{2 r}} \sum_{i=n+1}^{\infty}\left[\frac{1}{(i+1)^{2 r}}-\frac{1}{i^{2 r}}\right] S_{i, n}(\Upsilon) \\
& +\frac{1}{(m+1)^{2 r}} \frac{1}{(n+1)^{2 r}} S_{m, n}(\Upsilon) \|_{p, \omega} \\
& \leq \sum_{i=m+1}^{\infty} \sum_{j=n+1}^{\infty}\left(\frac{1}{i^{2 r}}-\frac{1}{(i+1)^{2 r}}\right)\left(\frac{1}{j^{2 r}}-\frac{1}{(j+1)^{2 r}}\right)\left\|S_{i, j}(\Upsilon)\right\|_{p, \omega}+ \\
& +\frac{1}{(m+1)^{2 r}} \sum_{j=n+1}^{\infty}\left[\frac{1}{j^{2 r}}-\frac{1}{(j+1)^{2 r}}\right]\left\|S_{m, j}(\Upsilon)\right\|_{p, \omega}+\frac{1}{(n+1)^{2 r}} \times
\end{aligned}
$$




$$
\begin{gathered}
\times \sum_{i=m+1}^{\infty}\left[\frac{1}{i^{2 r}}-\frac{1}{(i+1)^{2 r}}\right]\left\|S_{i, n}(\Upsilon)\right\|_{p, \omega}+\frac{1}{(m+1)^{2 r}} \frac{1}{(n+1)^{2 r}}\left\|S_{m, n}(\Upsilon)\right\|_{p, \omega} \\
\lesssim \frac{1}{(m+1)^{2 r}(n+1)^{2 r}}\|\Upsilon\|_{p, \omega}=\frac{1}{(m+1)^{2 r}(n+1)^{2 r}}\left\|g^{(2 r, 2 r)}\right\|_{p, \omega},
\end{gathered}
$$

and, hence,

$$
\begin{gathered}
Y_{m, n}(g)_{p, \omega}=Y_{m, n}\left(g-S_{m, \circ}(g)-S_{\circ, n}(g)+S_{m, n}(g)\right)_{p, \omega} \\
\leq\left\|g-S_{m, \circ}(g)-S_{\circ, n}(g)+S_{m, n}(g)\right\|_{p, \omega} \lesssim \frac{1}{(m+1)^{2 r}(n+1)^{2 r}}\left\|g^{(2 r, 2 r)}\right\|_{p, \omega}
\end{gathered}
$$

which completes the proof.

Here we give the proof of Potapov type Theorem 1.2.

Proof of Theorem 1.2. For $r \in \mathbb{N}$, this was obtained in [5]:

$$
Y_{m, n}(f)_{p, \omega} \leq C_{p, r,[\omega]_{A_{p}}} \Omega_{r}\left(f, \frac{1}{m}, \frac{1}{n}\right)_{p, \omega} .
$$

We suppose that $r \in \mathbb{R}^{+} \backslash \mathbb{N}$. For $0 \leq \alpha \leq \beta \leq 1$

$\int_{\mathbb{T}}\left|\left[I-\sigma_{h, \circ}\right]^{\beta} f(x, y)\right|^{p} \omega(x, y) d x \lesssim \int_{\mathbb{T}}\left|\left[I-\sigma_{h, \circ}\right]^{\alpha} f(x, y)\right|^{p} \omega(x, y) d x, \quad$ for almost every $y$, $\int_{\mathbb{T}}\left|\left[I-\sigma_{\circ, k}\right]^{\beta} f(x, y)\right|^{p} \omega(x, y) d x \lesssim \int_{\mathbb{T}}\left|\left[I-\sigma_{\circ, k}\right]^{\alpha} f(x, y)\right|^{p} \omega(x, y) d x, \quad$ for almost every $x$, were proved in $[1,(2.5)]$. The same proof also holds for $0 \leq \alpha \leq \beta<\infty$. Hence

$$
\Omega_{\beta}(f, \cdot,)_{p, \omega} \lesssim \Omega_{\alpha}(f, \cdot, \cdot)_{p, \omega} .
$$

From (4.1) and the last inequality we have

$$
Y_{m, n}(f)_{p, \omega} \leq c \Omega_{[r]+1}\left(f, \frac{1}{m}, \frac{1}{n}\right)_{p, \omega} \leq C \Omega_{r}\left(f, \frac{1}{m}, \frac{1}{n}\right)_{p, \omega}, \quad m, n \in \mathbb{N} .
$$

Proof of Corollary 3.3. Let $r \in \mathbb{R}^{+}$and $p \in(1, \infty)$. Suppose that $\omega \in A_{p}(\mathbb{T}), T \in \mathfrak{U}_{n}$ and $0<h<\pi /(n+1)$. From one dimensional inequality $[1,2]$

$$
\left\|\left[I-\sigma_{h}\right]^{r} T\right\|_{L_{\omega}^{p}} \lesssim(n+1)^{-2 r}\left\|T^{(2 r)}\right\|_{L_{\omega}^{p}}
$$

we obtain

$$
\int_{\mathbb{T}}\left|\left[I-\sigma_{h}\right]^{r} T(x)\right|^{p} \omega(x) d x \lesssim(n+1)^{-2 r p} \int_{\mathbb{T}}\left|T^{(2 r)}(x)\right|^{p} \omega(x) d x .
$$

From (4.2), we have for almost every $y$

$$
\int_{\mathbb{T}}\left|\left[I-\sigma_{h, o}\right]^{r} T_{1}(x, y)\right|^{p} \omega(x, y) d x \lesssim(m+1)^{-2 r p} \int_{\mathbb{T}}\left|T_{1}^{(2 r, o)}(x, y)\right|^{p} \omega(x, y) d x .
$$

Then

$$
\begin{gathered}
\int_{\mathbb{T}^{2}}\left|\left[I-\sigma_{h, \circ}\right]^{r} T_{1}(x, y)\right|^{p} \omega(x, y) d x d y \lesssim(m+1)^{-2 r p} \int_{\mathbb{T}^{2}}\left|T_{1}^{(2 r, \circ)}(x, y)\right|^{p} \omega(x, y) d x d y, \\
\Omega_{r}\left(T_{1}, \pi /(m+1), \cdot\right)_{p, \omega} \lesssim(m+1)^{-2 r}\left\|T_{1}^{(2 r, \circ)}\right\|_{p, \omega}
\end{gathered}
$$

holds. Other inequalities can be proved by the same method. 
Proof of Theorem 1.4 . For $\delta, \xi>0$, there exist natural numbers $m, n$ so that $\frac{m}{\pi} \leq$ $1 / \delta<2 \frac{m}{\pi}, \quad \frac{n}{\pi} \leq 1 / \xi<2 \frac{n}{\pi}$. Setting

$$
\Theta_{m, n}(f):=S_{m, \circ}(f)-S_{\circ, n}(f)+S_{m, n}(f),
$$

from Theorem 1.2 one can get

$$
\begin{gathered}
A_{1}=\left\|f-S_{m, \circ}(f)-S_{\circ, n}(f)+S_{m, n}(f)\right\|_{p, \omega} \\
\lesssim Y_{m, n}(f)_{p, \omega} \lesssim \Omega_{r}\left(f, m^{-1}, n^{-1}\right)_{p, \omega} \lesssim \Omega_{r}\left(f, \pi m^{-1}, \pi n^{-1}\right)_{p, \omega} .
\end{gathered}
$$

Secondly, we set $A_{2}=\left\|S_{m, \circ}^{(2 r, \circ)}\left(f-S_{\circ, n}(f)\right)\right\|_{p, \omega}$ and $\gamma=f-S_{\circ, n}(f)$. By one dimensional inequality (see Lemma 2.10)

$$
\delta^{2 r}\left(\int_{\mathbb{T}}\left|T_{m}^{(2 r)}(x)\right|^{p} \omega(x) d x\right)^{1 / p} \lesssim\left(\int_{\mathbb{T}}\left|\left[T_{m}(x)-\sigma_{\frac{1}{m}} T_{m}(x)\right]^{r}\right|^{p} \omega(x) d x\right)^{1 / p}
$$

we get, for almost every $y$,

$$
\begin{gathered}
\delta^{2 r}\left(\int_{\mathbb{T}}\left|S_{m, \circ}^{(2 r, \circ)}(\gamma)\right|^{p} \omega(x, y) d x\right)^{1 / p} \lesssim\left(\int_{\mathbb{T}}\left|\nabla_{\frac{1}{m}, \circ}^{r, \circ} S_{m, \circ}(\gamma)\right|^{p} \omega(x, y) d x\right)^{1 / p}, \quad \text { and } \\
\delta^{2 r p} \int_{\mathbb{T}}\left|S_{m, \circ}^{(2 r, \circ)}(\gamma)\right|^{p} \omega(x, y) d x \lesssim \int_{\mathbb{T}}\left|\nabla_{\frac{1}{m}, \circ}^{r, \circ} S_{m, \circ}(\gamma)\right|^{p} \omega(x, y) d x .
\end{gathered}
$$

Then

$$
\begin{gathered}
\delta^{2 r p} \int_{\mathbb{T}^{2}}\left|S_{m, \circ}^{(2 r, \circ)}(\gamma)\right|^{p} \omega(x, y) d x d y \lesssim \int_{\mathbb{T}^{2}}\left|\nabla_{\frac{1}{m}, \circ}^{r, \circ} S_{m, \circ}(\gamma)\right|^{p} \omega(x, y) d x d y, \quad \text { and } \\
\delta^{2 r} A_{2} \lesssim\left\|\nabla_{\frac{1}{m}, \circ}^{r, \circ} S_{m, \circ}(\gamma)\right\|_{p, \omega}=\left\|S_{m, \circ}\left(\nabla_{\frac{1}{m}, \circ}^{r, \circ} \gamma\right)\right\|_{p, \omega} \\
\lesssim\left\|\nabla_{\frac{1}{m}, \circ}^{r, \circ} \gamma\right\|_{p, \omega}=\left\|\nabla_{\frac{1}{m}, \circ}^{r, \circ}\left(f-S_{\circ, n}(f)\right)\right\|_{p, \omega}=\left\|\nabla_{\frac{1}{m}, \circ}^{r, \circ} f-\nabla_{\frac{1}{m}, \circ}^{r, 0} S_{\circ, n}(f)\right\|_{p, \omega} \\
=\left\|\nabla_{\frac{1}{m}, \circ}^{r, \circ} f-S_{0, \circ}\left(\nabla_{\frac{1}{m}, \circ}^{r, \circ} f\right)-S_{\circ, n}\left(\nabla_{\frac{1}{m}, \circ}^{r, \circ} f\right)+S_{0, n}\left(\nabla_{\frac{1}{m}, \circ}^{r, \circ} f\right)\right\|_{p, \omega} \\
\qquad Y_{0, n}\left(\nabla_{\frac{1}{m}, \circ}^{r, \circ} f\right)_{p, \omega} \lesssim \Omega_{r}\left(\nabla_{\frac{1}{m}, \circ}^{r, \circ} f, 1, \frac{1}{n}\right)_{p, \omega} \\
=\sup _{0 \leq h \leq 1}\left\|\nabla_{h, \circ}^{r, \circ} \nabla_{\circ, k}^{\circ, r}\left(\nabla_{\frac{1}{m}, \circ}^{r, \circ} f\right)\right\|_{p, \omega} \lesssim \sup _{0 \leq k \leq 1 / n}\left\|\nabla_{\circ, k}^{\circ, r}\left(\nabla_{\frac{1}{m}, \circ}^{r, \circ} f\right)\right\|_{p, \omega} \\
\qquad \leq \leq k \leq 1 / n \\
\sup _{\substack{0 \leq h \leq 1 / m \\
0 \leq k \leq 1 / n}}\left\|\nabla_{h, \circ}^{r, \circ}\left(\nabla_{\circ, k}^{\circ, r} f\right)\right\|_{p, \omega}=\Omega_{r}\left(f, \frac{1}{m}, \frac{1}{n}\right)_{p, \omega} \lesssim \Omega_{r}\left(f, \frac{\pi}{m}, \frac{\pi}{n}\right)_{p, \omega} .
\end{gathered}
$$

Taking

$$
A_{3}:=\left\|S_{\circ, n}^{(\circ, 2 r)}\left(f-S_{m, \circ}(f)\right)\right\|_{p, \omega} \text { and } A_{4}:=\left\|S_{m, n}^{(2 r, 2 r)}(f)\right\|_{p, \omega},
$$

similar arguments give us

$$
\begin{aligned}
& \xi^{2 r} A_{3} \lesssim\left\|S_{\circ, n}^{(\circ, 2 r)}\left(f-S_{m, \circ}(f)\right)\right\|_{p, \omega} \lesssim \Omega_{r}\left(f, \pi m^{-1}, \pi n^{-1}\right)_{p, \omega} \\
& \delta^{2 r} \xi^{2 r} A_{4} \lesssim\left\|S_{m, n}^{(2 r, 2 r)}(f)\right\|_{p, \omega} \lesssim \Omega_{r}\left(f, \pi m^{-1}, \pi n^{-1}\right)_{p, \omega}, \text { and } \\
& A_{1}+\delta^{2 r} A_{2}+\xi^{2 r} A_{3}+\delta^{2 r} \xi^{2 r} A_{4} \lesssim \Omega_{r}(f, \delta, \xi)_{p, \omega} .
\end{aligned}
$$


Definition of $K$-functional gives

$$
K(f, \delta, \xi, p, \omega, 2 r) \leq A_{1}+\delta^{2 r} A_{2}+\xi^{2 r} A_{3}+\delta^{2 r} \xi^{2 r} A_{4} \lesssim \Omega_{r}(f, \delta, \xi)_{p, \omega} .
$$

Consider reverse of the last inequality. For any $g_{1} \in W_{p, \omega}^{r, o}, g_{2} \in W_{p, \omega}^{\circ, s}, g \in W_{p, \omega}^{r, s}$ we have

$$
\begin{gathered}
\Omega_{r}(f, \delta, \xi)_{p, \omega} \leq \Omega_{r}\left(f-g_{1}-g_{2}-g, \delta, \xi\right)_{p, \omega}+\Omega_{r}\left(g_{1}, \delta, \xi\right)_{p, \omega}+ \\
+\Omega_{r}\left(g_{2}, \delta, \xi\right)_{p, \omega}+\Omega_{r}(g, \delta, \xi)_{p, \omega} \lesssim\left\|f-g_{1}-g_{2}-g\right\|_{p, \omega}+ \\
\quad+\delta^{2 r}\left\|g_{1}^{(2 r, o)}\right\|_{p, \omega}+\xi^{2 r}\left\|g_{2}^{(\circ, 2 r)}\right\|_{p, \omega}+\delta^{2 r} \xi^{2 r}\left\|g^{(2 r, 2 r)}\right\|_{p, \omega} .
\end{gathered}
$$

From the last inequality, taking infimum on $g_{1} \in W_{p, \omega}^{r, \circ}, g_{2} \in W_{p, \omega}^{\circ, s}, g \in W_{p, \omega}^{r, s}$, one gets

$$
\Omega_{r}(f, \delta, \xi)_{p, \omega} \lesssim K(f, \delta, \xi, p, \omega, 2 r) .
$$

Proof of Theorem 1.6. Using the properties of $\Omega_{r}(f, \cdot, \cdot)_{p, \omega}$ we have

$$
\begin{gathered}
\Omega_{r}\left(f, \frac{1}{m}, \frac{1}{n}\right)_{p, \omega} \leq \Omega_{r}\left(f-W_{2^{\mu}, 2^{\nu}} f, \frac{1}{m}, \frac{1}{n}\right)_{p, \omega}+\Omega_{r}\left(W_{2^{\mu}, 2^{\nu}} f, \frac{1}{m}, \frac{1}{n}\right)_{p, \omega}, \text { and } \\
\Omega_{r}\left(f-W_{2^{\mu}, 2^{\nu}} f, \frac{1}{m}, \frac{1}{n}\right)_{p, \omega} \lesssim\left\|f-W_{2^{\mu}, 2^{\nu}}\right\|_{p, \omega} \lesssim Y_{2^{\mu}, 2^{\nu}}(f)_{p, \omega} .
\end{gathered}
$$

By the property

$$
\begin{gathered}
W_{2^{\mu}, 2^{\nu}} f-W_{0,0} f \leq \sum_{i=0}^{\mu}\left(W_{2^{i}, 2^{\nu}} f-W_{\left\lfloor 2^{i-1}\right\rfloor, 2^{\nu}} f\right)+\sum_{j=0}^{\nu}\left(W_{2^{\mu}, 2^{j}} f-W_{2^{\mu},\left\lfloor 2^{j-1}\right\rfloor} f\right)- \\
-\sum_{i=0}^{\mu} \sum_{j=0}^{\nu} W_{2^{i}, 2^{j}} f-W_{2^{i},\left\lfloor 2^{j-1}\right\rfloor} f-W_{\left\lfloor 2^{i-1}\right\rfloor, 2^{j}} f+W_{\left\lfloor 2^{i-1}\right\rfloor,\left\lfloor 2^{j-1}\right\rfloor} f,
\end{gathered}
$$

we find (see Lemma 2.12 for quantities $\left.\psi_{i, \nu}(f), h_{\mu, j}(f), \varphi_{i, j}(f)\right)$

$$
\begin{gathered}
\Omega_{r}\left(W_{2^{\mu}, 2^{\nu}} f, \frac{1}{m}, \frac{1}{n}\right)_{p, \omega}=\Omega_{r}\left(W_{2^{\mu}, 2^{\nu}} f-W_{0,0} f, \frac{1}{m}, \frac{1}{n}\right)_{p, \omega} \\
\leq \sum_{i=0}^{\mu} \Omega_{r}\left(\psi_{i, \nu}(f), \frac{1}{m}, \frac{1}{n}\right)_{p, \omega}+\sum_{j=0}^{\nu} \Omega_{r}\left(h_{\mu, j}(f), \frac{1}{m}, \frac{1}{n}\right)_{p, \omega}+ \\
+\sum_{i=0}^{\mu} \sum_{j=0}^{\nu} \Omega_{r}\left(\varphi_{i, j}(f), \frac{1}{m}, \frac{1}{n}\right)_{p, \omega} \\
\lesssim \frac{1}{m^{2 r}} \sum_{i=0}^{\mu}\left\|\left(\psi_{i, \nu}(f)\right)^{(2 r, \circ)}\right\|_{p, \omega}+\frac{1}{n^{2 r}} \sum_{j=0}^{\nu}\left\|\left(h_{\mu, j}(f)\right)^{(\circ, 2 r)}\right\|_{p, \omega}+ \\
+\frac{1}{m^{2 r}} \frac{1}{n^{2 r}} \sum_{i=0}^{\mu} \sum_{j=0}^{\nu}\left\|\left(\varphi_{i, j}(f)\right)^{(2 r, 2 r)}\right\|_{p, \omega} \lesssim \\
\lesssim \frac{1}{m^{2 r}} \sum_{i=0}^{\mu} 2^{2 r i} Y_{\left\lfloor 2^{i-1}\right\rfloor, 2^{j}}(f)_{p, \omega}+\frac{1}{n^{2 r}} \sum_{j=0}^{\nu} 2^{2 r j} Y_{2^{i},\left\lfloor 2^{j-1}\right\rfloor}(f)_{p, \omega}+ \\
+\frac{1}{m^{2 r}} \frac{1}{n^{2 r}} \sum_{i=0}^{\mu} \sum_{j=0}^{\nu} 2^{2 i r+2 r j} Y_{\left.\left\lfloor 2^{i-1}\right\rfloor, 2^{j-1}\right\rfloor}(f)_{p, \omega} .
\end{gathered}
$$

Suppose that $m, n$ satisfy $2^{\mu} \leq m<2^{\mu+1}, 2^{\nu} \leq n<2^{\nu+1}$. Then, one can get

$$
\Omega_{r}\left(f, \frac{1}{m}, \frac{1}{n}\right)_{p, \omega} \lesssim \frac{1}{m^{2 r}} \frac{1}{n^{2 r}} \sum_{i=0}^{\mu} \sum_{j=0}^{\nu} 2^{2 r i+2 r j} Y_{\left\lfloor 2^{i-1}\right\rfloor,\left\lfloor 2^{j-1}\right\rfloor}(f)_{p, \omega}
$$




$$
\begin{gathered}
\lesssim \frac{1}{m^{2 r} n^{2 r}} \sum_{i=0}^{\mu+1} \sum_{j=0}^{\nu+1} 2^{2 r i+2 r j} Y_{\left\lfloor 2^{i-1}\right\rfloor,\left\lfloor 2^{j-1}\right\rfloor}(f)_{p, \omega} \\
\lesssim \frac{1}{m^{2 r} n^{2 r}} \sum_{i=0}^{m} \sum_{j=0}^{n}[(i+1)(j+1)]^{2 r-1} Y_{i, j}(f)_{p, \omega} .
\end{gathered}
$$

Acknowledgment. The author is indebted to referees for valuable suggestions.

\section{References}

[1] R. Akgün, Polynomial approximation in weighted Lebesgue spaces, East J. Approx. 17(3), 253-266, 2011.

[2] R. Akgün, Approximating polynomials for functions of weighted Smirnov-Orlicz spaces, J. Funct. Spaces Appl. 2012, 1-41, ID 982360, 2012.

[3] R. Akgün, Realization and characterization of modulus of smoothness in weighted Lebesgue spaces, St. Petersburg Math. J. 26 (5), 741-756, 2015.

[4] R. Akgün, Mixed modulus of continuity in Lebesgue spaces with Muckenhoupt weights, Turkish J. Math. 40 (6), 1169-1192, 2016.

[5] R. Akgün, Mixed modulus of smoothness with Muckenhoupt weights and approximation by angle, Complex Var. Elliptic Equ. 64 (2), 330-351, 2019.

[6] R. Akgün, Nikol'ski, Jackson and Ul'yanov type inequalities with Muckenhoupt weights, arXiv:1709.02928 [math.CA].

[7] P.L. Butzer, H. Dyckhoff, E. Görlich, and R.L. Stens, Best trigonometric approximation, fractional order derivatives and Lipschitz classes, Canad. J. Math. 29 (4), 781-793, 1977.

[8] C. Cottin, Mixed K-functionals: a measure of smoothness for blending-type approximation, Math. Z. 204 (1), 69-83, 1990.

[9] E. DiBenedetto, Real Analysis, Second Ed., Birkhäuser, Boston, 2016.

[10] A. Guven and D.M. Israfilov, Improved inverse theorems in weighted Lebesgue and Smirnov spaces, Bull. Belg. Math. Soc. Simon Stevin 14 (4), 681-692, 2007.

[11] A. Guven and V. Kokilashvili, On the mean summability by Cesaro method of Fourier trigonometric series in two-weighted setting, J. Inequal. Appl. 2006, Art. 41837, 2006.

[12] D.M. Israfilov, Approximation by p-Faber polynomials in the weighted Smirnov class $E^{p}(G, \omega)$ and the Bieberbach polynomials, Constr. Approx. 17 (3), 335-351, 2001.

[13] S.Z. Jafarov, On moduli of smoothness in Orlicz classes, Proc. Inst. Math. Mech. Natl. Acad. Sci. Azerb. 33, 95-100, 2010.

[14] V. Kokilashvili and Y.E. Yildirir, On the approximation in weighted Lebesgue spaces, Proc. A. Razmadze Math. Inst. 143, 103-113, 2007.

[15] A. D. Nakhman and B. P. Osilenker, Estimates of weighted norms of some operators generated by multiple trigonometric Fourier series. (Russian) Izv. Vyssh. Uchebn. Zaved. Mat. 26 (4), 39-50, 1982.

[16] M.K. Potapov, Approximation by "angle" (in Russian). In: Proceedings of the Conference on the Constructive Theory of Functions and Approximation Theory, Budapest, 1969, Akadémiai Kiadó, pp. 371-399, 1972.

[17] M.K. Potapov, The Hardy-Littlewood and Marcinkiewicz-Littlewood-Paley theorems, approximation "by an angle", and the imbedding of certain classes of functions (in Russian), Mathematica (Cluj) 14 (37), 339-362, 1972.

[18] M.K. Potapov, A certain imbedding theorem (in Russian), Mathematica (Cluj) 14(37), 123-146, 1972.

[19] M.K. Potapov, Approximation "by angle", and imbedding theorems (in Russian), Math. Balkanica 2, 183-198, 1972. 
[20] M.K. Potapov, Imbedding of classes of functions with a dominating mixed modulus of smoothness (in Russian), Trudy Mat. Inst. Steklov. 131, 199-210, 1974.

[21] M.K. Potapov and B.V. Simonov, On the relations between generalized classes of Besov-Nikolskiu and Weyl-Nikolskiu functions, Proc. Steklov Inst. Math. 214 (3), 243259, 1996.

[22] M.K. Potapov, B.V. Simonov, and B. Lakovich, On estimates for the mixed modulus of continuity of a function with a transformed Fourier series, Publ. Inst. Math. (Beograd) (N.S.) 58 (72), 167-192, 1995.

[23] M.K. Potapov, B.V. Simonov, and S.Y. Tikhonov, Embedding theorems for BesovNikolskiu and Weyl-Nikolskiu classes in a mixed metric, Moscow Univ. Math. Bull. 59, 19-26, 2005.

[24] M.K. Potapov, B.V. Simonov and S.Y. Tikhonov, Transformation of Fourier series using power and weakly oscillating sequences, Math. Notes 77 (1-2), 90-107, 2005.

[25] M.K. Potapov, B.V. Simonov, and S.Y. Tikhonov, Relations between mixed moduli of smoothness and embedding theorems for the Nikolskir classes, Proc. Steklov Inst. Math. 269 (1), 197-207, 2010.

[26] M.K. Potapov, B.V. Simonov, and S.Y. Tikhonov, Mixed moduli of smoothness in $L_{p}, 1<p<\infty$ : A survey, Surv. Approx. Theory 8, 1-57, 2013.

[27] S.G. Samko, A.A. Kilbas, and O.I. Marichev, Fractional Integrals and Derivatives. Theory and Applications, Translated from Russian 1987, Gordon and Breach Science Publishers, Yverdon, 1993.

[28] R. Taberski, Differences, moduli and derivatives of fractional orders, Comment. Math. Prace Mat. 19 (2), 389-400, 1976/77.

[29] Y.E. Yildirir and D.M. Israfilov, Approximation theorems in weighted Lorentz spaces, Carpathian J. Math. 26 (1), 108-119, 2010. 